\title{
The right organ for the right recipient: the Ninth Annual American Society of Transplant Surgeons' State-of-the-Art Winter Symposium
}

Sung RS, Abt PL, Desai DM, Garvey CA, Segev DL, Kaufman DB. The right organ for the right recipient: the Ninth Annual American Society of Transplant Surgeons' State-of-the-Art Winter Symposium.

Clin Transplant 2011: 25: E592-E598. (C) 2011 John Wiley \& Sons A/S.

Abstract: With an increasing number of individuals with end-stage organ disease and the increasing success of organ transplantation, the demand for transplants has steadily increased. This growth has led to a greater need to utilize organs from as many donors as possible. As selection criteria have become less stringent to accommodate increasing demand, transplant outcomes are more strongly influenced by recipient and donor factors; thus, finding the right organ for the right recipient is more important than ever. The Ninth Annual American Society of Transplant Surgeons (ASTS) State-of-the-Art Winter Symposium, entitled "The Right Organ for the Right Recipient," addressed the matching of donor organs to appropriate recipients. Representative dilemmas in the matching of donor organs with recipients were discussed. These included the following: matching by donor and recipient risk characteristics; use of organs with risk for disease transmission; biologic incompatibility; use of organs from donors after cardiac death; the justification for combined organ transplants like liver-kidney and kidney-pancreas; and the role of allocation in facilitating the matching of donors and recipients. Regardless of the particular issue, decisions about donor-recipient matching should be evidence-based, practical, and made with the goal of maximizing organ utilization while still protecting individual patient interests.

\section{Randall S. Sung ${ }^{a}$, Peter L. Abt ${ }^{b}$, Dev M. Desai ${ }^{\mathrm{C}}$, Catherine A. Garvey $^{\mathrm{d}}$, Dorry L. Segev ${ }^{\mathrm{e}}$ and Dixon B. Kaufman ${ }^{f}$}

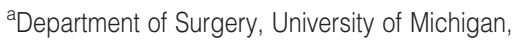
Ann Arbor, MI, 'D Department of Surgery, University of Pennsylvania, Philadelphia, PA, 'Department of Surgery, University of Texas Southwestern, Dallas, TX, ${ }^{\mathrm{d}}$ The Transplant Center, University of Minnesota Medical CenterFairview, Minneapolis, MN, eDepartment of Surgery, Johns Hopkins University, Baltimore MD and 'Department of Surgery, University of Wisconsin, Madison, WI, USA

Key words: organ allocation - organ donation organ transplantation - recipient selection transplant surgery

Corresponding author: Randall S. Sung, MD, Associate Professor of Surgery, Department of Surgery, Section of Transplantation, University of Michigan Health System, 1500 E Medical Center Drive, 2922 Taubman, Ann Arbor, Ml 481095331, USA.

Tel.: +1 734936 8363; fax: +1 734763 3187; e-mail: rssung@umich.edu

Conflict of interest: None of the authors, or their family members, has a financial interest in a business or commercial entity, or an intellectual property interest, that relates to this manuscript.

Accepted for publication 24 May 2011
Organ transplant outcomes have improved over the past several decades. Over this same time period, the number of individuals with end-stage organ failure who are potential transplant candidates has rapidly increased. This increased demand has been augmented by the expansion of recipient selection criteria, particularly with respect to age and other medical comorbidities. The resulting increase in disparity between the numbers of candidates and the supply of donors has led to the desire to utilize organs from as many reasonable organ donors as possible, including the use of organs from marginal donors. As the boundaries of both donor and recipient selection have expanded, we are learning that outcomes are being more heavily influenced by particular recipient and 
donor characteristics and that certain types of organs may be more suitable for certain types of recipients. The Ninth Annual American Society of Transplant Surgeons (ASTS) State-of-the-Art Winter Symposium, entitled "The Right Organ for the Right Recipient," addressed the matching of donor organs to appropriate recipients.

A variety of topics were addressed that reflect current issues in the assignment of donor organs and recipients. These includes the following: matching by donor and recipient risk characteristics; use of organs with risk for disease transmission; biologic incompatibility; the use of organs from donors after cardiac death (DCD); the justification for the use of combined organs like liver-kidney and kidney-pancreas; and the role of allocation in facilitating donor-recipient matching.

\section{Historical perspectives}

William Pfaff, a former president of the Southeastern Regional Organ Procurement Foundation (SEOPF) and the United Network for Organ Sharing (UNOS), described the early days of organ allocation in the lecture, "Youthful Indiscretions: Initial Attempts to Match Donors and Recipients." Organ sharing dates back to 1969 with the original eight members of SEOPF. The early directors were heads of transplant programs, most of whom were surgeons. Later, membership would include Organ Procurement Organizations (OPOs) and histocompatibility laboratories that served multiple programs. Decisions were largely by consensus, and participation in sharing was voluntary. While sharing was very haphazard in the 1970s, as database capabilities and membership increased, sharing became a mandatory condition of membership - this was not welcomed by all OPOs at the time.

The scientific reports generated as a result of increased database capabilities were an important part of the growth of transplantation in the early to mid-1980s. Some of the early observations formed the basis for allocation for many years: variation in OPO and center outcomes, the modest effect of matching, the positive effect of sharing highly matched transplants, and the effect of cold ischemia times (CITs). SEOPF also introduced quality indicators in histocompatibility laboratories. In 1984, following the enactment of the National Organ Transplantation Act (NOTA), SEOPF, as UNOS, applied for and received the federal contract for the Organ Procurement and Transplantation Network (OPTN). In retrospect, as Dr. Pfaff stated, "We were only partly literate, the languages of histocompatibility and transplant immunology were incompletely formed. Newborn, we were naïve, but learned collectively and separately by trial and error. I think we made reasonable rules as went along and as new tools and actions were contemplated. Our success was the consequence of altruistic collaboration."

\section{Donors with high risk for disease transmission}

With any organ transplant, the risk of transmission of infections or malignancy is well recognized. There are many deleterious effects if certain diseases in the organ donor are transmitted to an immunosuppressed transplant recipient. The OPTN has screening policies that require all donors to undergo serologic testing for common diseases and diseases at high risk of transmission. The well-known infections are syphilis and the viral diseases cytomegalovirus, Epstein-Barr virus, hepatitis $\mathrm{C}$, hepatitis $\mathrm{B}$, the human immunodeficiency virus (HIV), and human T-lymphotropic viruses 1 and 2 . Other viral diseases such as West Nile, lymphocytic choriomeningits, and rabies have been transmitted by organ transplantation, so an accurate history and clinical acumen are important in minimizing risk. Recipients are also at risk for transmission of donor fungal infections such as coccidiomycosis or histoplasmosis. Mycobacterium tuberculosis and parasites including toxoplasma gondi and Chagas (Trypansoma cruzi) have been transmitted.

While extensive donor screening processes have been developed, the potential for the transmission still exists. Most of the data obtained regarding the medical and social history of donors come from next of kin, which may be suspect in its accuracy and completeness. The availability of screening tests varies around the country. Screening methods and procedures, while highly accurate, are not perfect. False positive tests may lead to discarding organs safe for transplants, and false negative or absent testing of very rare diseases may result in disease transmission. In the case of a deceased donor, especially for either unstable donors or for organs unable to tolerate cold ischemia, time constraints may dictate that a decision to transplant be made without complete screening information. When testing a living donor, more time is available, and a complete donor history and screening can be completed.

Although the risk of disease transmission is low in thoroughly screened donors, it is prudent that all recipients of organs must be informed of the risk of transmission of infection or malignancy with any organ transplant. This information should be shared with transplant candidates early in the 
referral process and included in educational materials. This risk discussion continues up to and including the time of transplant.

In the Keynote debate, entitled "Tell Her About It: Do Recipients of High-Risk Organs Require Complete Informed Consent?," the ethics of using organs from high-risk donors and the degree to which recipients should be informed of the risks were discussed. ASTS President John Roberts stated that, according to the American Medical Association (AMA) Code of Medical Ethics in 1947, patients should hold that "obedience to the prescriptions of his physician should be prompt and implicit" and that "he should never permit his own crude opinions as to their fitness to influence his attention to them." As the medical environment evolved to acknowledge the premise of self-determination, the issue of the extent of physician disclosure emerged. The Reasonable Physician standard, by which responsibility for disclosure" is limited to those disclosures which a reasonable medical practitioner would make under the same or similar circumstances," gave way to the Reasonable Patient Standard. By 1981, the AMA Code of Ethics stated, "the patient's right of self-decision can be effectively exercised only if the patient possesses enough information to make an intelligent choice."

In transplant situations where consent may be required for certain types of organs (such as expanded criteria donor kidneys or those from high-risk donors as defined by the Centers for Disease Control [CDC]), the standard should be to disclose what a reasonable patient would want to know about the donor. Under this standard, what might be considered reasonable may change with the circumstances of the recipient (e.g., model for end-stage liver disease (MELD) score, anticipated time to transplant). Dr. Roberts emphasized that informed consent is an ongoing interactive process, especially when emotions present at the time of offer may obscure the patient's decision-making processes. He also pointed out that, contrary to some fears, informed consent does not decrease organ utilization (1).

Michael Abecassis, taking the opposite view, countered that paternalism has a greater place in decision making than is currently appreciated. The ability to comprehend the issues and assimilate the information required for effective decision making is extremely uncommon in patients. Patients frequently follow the recommendations of their physicians, and trust in the physician plays a greater role in patients' treatment decisions compared with other factors such as transparency, outcomes, efficiency, and value. Numerous research studies have indicated that patients typically perceive that stated risks do not apply to them, decisions are influenced by the way information is presented, and very little of presented information is retained. All of these findings suggest an important role for an active physician role in medical decision making.

The case of HIV transmission to organ transplant recipients has raised new issues of informed consent (2): Should potential recipients be informed only about the general risks associated with transplantation or also those specifically associated with an identified organ? Should the risks engendered by the behavior of donors be treated differently from those associated with the medical profiles of donors? Finally, is the supply of transplantable organs a singular public good to be distributed to maximize public health or is it a market of intermittently available goods from which eligible recipients might select to maximize their own well-being? The concept of a standard prospective consent for non-standard organs was proposed. Complete disclosure would be provided to those who ask for it, but would not be routine. There would be exceptions for certain recipient groups, the definition of "non-standard" organ could change over time, and mechanisms to accommodate for change in recipient status would exist.

\section{Crossing antibody barriers}

The sensitized kidney candidate is an important example of donor-recipient matching, as the choice of donor can have dramatic effects on the outcome of an individual recipient. James Gloor (Mayo Clinic) provided an overview of antibody detection technology. Traditional assays are cell-based and include, in increasing order of sensitivity, complement-dependent cytotoxic (CDC) crossmatch, AHG-augmented CDC cytotoxic crossmatch, and flow cytometric crossmatch. Solid phase antihuman leukocyte antibody (HLA) detection assays are more sensitive than cell-based assays and are able to quantify specific anti-HLA antibody activity by mean fluorescence intensity (MFI). A "virtual crossmatch" attempts to use information from a solid phase assay that has defined known candidate anti-HLA antibodies to predict what would have occurred in a cell-based assay. While standardization among laboratories has been a concern, several studies suggest that solid phase assays are consistent between HLA laboratories in identifying strong and moderate strength antibodies, but quite variable in identifying weak (1000-2000 MFI) antibodies. A Mayo study 
demonstrated that patients with negative flow crossmatches can have Class I or II DSA in $7 \%$ and $20 \%$ of cases, respectively. The clinical significance of low-titer DSA detectable by solid phase assay in the setting of a negative flow crossmatch is somewhat unclear, although some evidence exists that those with DSA have a higher risk of antibody-mediated rejection than those without DSA (3).

There is a general correlation between DSA measured by solid phase assays and channel shift seen in flow cytometric crossmatches, although on a population level this correlation is relatively weak. However, for a given patient, this relationship is much more stable, and solid phase assays (which do not depend on donor cells) can be very useful in post-transplant monitoring. For a given patient, MFI trends in post-transplant antibody levels correlate strongly with trends in cell-based activity as well as histologic findings on protocol and for-cause biopsies (4).

Robert Montgomery (Johns Hopkins) then reviewed kidney transplantation across anti-HLA barriers. Highly sensitized patients in the United States have extremely low rates of living or deceased donor kidney transplantation. At Hopkins, two modalities are offered to patients with incompatible living donors: desensitization with plasmapheresis and low-dose intravenous immunoglobulin, or kidney paired donation (KPD). Results from desensitization at this center are encouraging: of 187 patients who started the HLA desensitization protocol between 1998 and 2009, $97 \%$ achieved transplantation, with a 10 -yr deathcensored graft survival around $90 \%$. Five-yr deathcensored graft survival of ABO incompatible transplants between 1999 and 2007 was also around 90\%.

Furthermore, a striking survival advantage was seen when comparing those who underwent positive HLA crossmatch $(+\mathrm{XM})$ kidney transplantation at Johns Hopkins with matched controls from the UNOS waiting list (regardless of whether those matched controls were ultimately transplanted).

However, there are conflicting pressures driving innovation in this field: pressures to address vulnerable populations and take risks to achieve transplantation vs. others to improve results and therefore limit risk. Evidence from the Hopkins $+\mathrm{XM}$ cohort suggests that risk adjustment models may not adequately account for the risk seen in $+\mathrm{XM}$ patients, and as such potentially restrict innovation at transplant centers seeking to develop and improve such programs. KPD offers a workaround to desensitization (5), or complements desensitization in cases of broadly sensitized patients who are unlikely to ever find a negative crossmatch (6).

\section{Combined transplants}

The use of combined transplants engenders debate because (i) criteria for candidacy are not clearly defined, and (ii) combined transplants potentially take a kidney from an arguably more suitable kidney-alone candidate. David Gerber (University of North Carolina) addressed appropriate candidates for simultaneous liver and kidney (SLK) transplantation. Comparing the pre-MELD to MELD era there has been a $41 \%$ increase in patients on dialysis and a $117 \%$ increase in SLK transplants. The decision as to who requires a combined transplant remains controversial. From the perspective of wait list mortality, liver transplant candidates on dialysis who are not listed for a kidney have the greatest risk of death, followed by SLK candidates on dialysis, and then SLK candidates not on dialysis. Lesser degrees of renal dysfunction correlate well with both unadjusted candidate waitlist mortality and post-transplant survival for liver transplant alone recipients (7).

While hepatorenal syndrome (HRS) is not usually an indication for SLK transplant, renal recovery is unpredictable and influenced by the presence of other perioperative conditions such as volume status, use of vasopressors, and calcineurin inhibitors. Pre-transplant dialysis in patients with HRS does not appear to impact survival compared to those with HRS who do not require dialysis. However, HRS is associated with inferior patient survival compared with recipients without HRS (8). Data were presented that demonstrated that the duration of pre-transplant creatinine elevation is predictive of renal function six and 12 months post-liver transplant (9).

Deceased simultaneous pancreas kidney (SPK) vs. pancreas after living donor kidney transplant should be viewed not as competing therapeutic options, but rather complementary to each other based upon patient preference, the availability of a living kidney donor, and access to deceased donor organs. Raja Kandaswamy from the University of Minnesota showed that patient survival from the time of kidney transplant for pancreas after living donor kidney is superior to SPK transplant $(99.5 \%$ vs. $95.1 \%$ at one yr). However, pancreas survival is greater with SPK transplant at one yr (84\% vs. $78 \%$ ) because of an increased risk of immunologic graft loss for pancreas after kidney transplants.

Distinct advantages of a pancreas after living donor kidney compared with SPK transplant 
include the inherent benefit of a living donor kidney transplant, no drain on the deceased donor pool, decreased surgical complications, shorter operative time, and possibly decreased waiting time for a pancreas transplant (10). The disadvantages include inferior pancreas survival compared with a SPK, two operations with increased expense, short-term adverse impact on renal function, and a second round of induction immunosuppression. In addition, organ availability may be more limited because of local allocation rules favoring use of SPK over solitary pancreas and because of more stringent donor selection for solitary pancreas compared with SPK. Given the high mortality associated with diabetes and endstage renal disease, priority should be given to obtaining a kidney for the potential recipient in as short a time as possible. Options include the following: living or deceased kidney transplant alone (KTA) or SPK transplant. For those considering KTA, recipients should be made aware of the option of pancreas after kidney transplant. Clearly, the patients' preferences in how they prioritize their interests in kidney and pancreas transplants are important to consider in planning the appropriate treatment. As a general guideline, a living donor kidney should be considered whenever possible, especially for recipients with long waits for deceased donor kidneys. If living donor is not an option, an SPK transplant or deceased donor KTA should be pursued with the option dependent on the candidate's comorbidities and preferences, and the transplant center's waiting time. Pancreas after kidney transplant is an important option among patients with excellent renal function and good health.

\section{Donor and recipient risk/outcomes}

Dorry Segev (Johns Hopkins) laid the statistical and epidemiological groundwork for matching donors with recipients, namely interaction or effect modification. Traditional regression models estimate the average association of one factor with another, while adjusting for other factors that act as confounders, so that an independent association can be demonstrated. An example might be that longer CIT is on average associated with worse survival after liver transplantation, independent of other factors that cause worse survival. Similarly, higher donor body mass index (BMI) is on average independently associated with worse survival. However, among patients who receive livers with short CIT, BMI seems to exert minimal effect, while among patients with longer CIT, those with higher donor BMI do substantially worse (11). Therefore, the effect of BMI is modified by CIT.

This effect modification can be studied in stratified models, where a different model is used for each stratum (category) of a suspected effect modifier; or in interaction term models, where in addition to the usual terms for the independent effects of each factor (such as one term for CIT and one for BMI), a term is introduced for the interaction between the factors (which would be interpreted as the change in the effect of BMI on survival for each unit change in CIT). The choice of model balances statistical efficiency, ease of interpretation of the coefficients, and underlying assumptions of the models. Regardless of methodology used, understanding effect modification can help in selecting the right donors for a given recipient's profile or in selecting the right target recipient group for a given donor (12).

Goran Klintmalm and Robert Merion debated the value of registry predictors and statistics compared with surgical judgment in donor-recipient matching. Dr. Klintmalm presented the limitations of registry data, which include the presence of incomplete and inaccurate data entry, inability to capture all relevant variables, and that many patients do not fit into artificially defined categories. The current MELD liver allocation system was offered as an example: as the implementation of MELD, the number of MELD exception categories and number of cases for review has continued to increase.

Dr. Merion argued that registry predictors, such as those used in the donor risk index (DRI) calculations derived for liver, kidney, and pancreas, are the most objective and reliable measures to match organs and recipients. For example, conventional surgical wisdom would suggest that high-risk organs should be utilized in low-risk recipients, as they would be better able to tolerate initial graft dysfunction; however, data from the Scientific Registry of Transplant Recipients demonstrated that these patients actually fared the worst from a transplant benefit perspective (13). Furthermore, Dr. Merion discussed the subjective issues around defining judgment, the injection of personal bias, and the limitations of experience in clinical decision making.

Donor and recipient matching in the high-risk donor (as defined by DRI) and DCD liver setting was addressed by Christopher Hughes from Emory University. Although data indicate that ischemic cholangiopathy, primary non-function, and graft failure are all more common among recipients of a DCD liver, more recent data indicate that acceptable outcomes can be achieved 
(14). Using a DCD risk index based upon five covariates (including prior transplant in the recipient, life support in the recipient, donor age, donor warm ischemic time, and cold ischemic time), it was demonstrated that organs with lowrisk scores had similar survival outcomes as transplants from brain-dead donors (15). Additional data from the Mayo Clinic Florida that included 108 DCD transplants revealed that DCD recipient survival at three yr was $88.1 \%$, comparable to $81.2 \%$ among 1400 donation after braindeath recipients. The rate of primary non-function among the DCD organs was $4.6 \%$ and that for ischemic cholangiopathy $7.4 \%$. Proper donor and recipient selection could lead to better outcomes with the use of DCDs; however, more data are needed to determine which recipients are at risk of developing cholangiopathy.

\section{Allocation}

While the appropriate matching of donors and recipients is often an issue encountered at the level of clinical practice, such matching is also within the purview of transplant policy. With that in mind, Mark Stegall (Mayo Clinic) and Mitchell Henry (Ohio State University) discussed the use of transplant benefit, as measured by life years from transplant (LYFT), in kidney allocation. To Dr. Stegall, the essential question to consider is whether to base any allocation system on transplant outcomes. Systems that would not utilize outcomes would be either random (lottery) or perhaps use waiting time; these are objective and might be perceived as fair and some would be very predictable.

Arguments in favor of outcome-based allocation include recognition of the fact that some patients do better than others with certain types of organs, utility (best use of organ), stewardship of the organ, preferences of donor families, and direction under the Final Rule. There are many examples of outcomes-based allocation in the current system, including the use of listing criteria, matching, crossmatching, and pediatric priority. While there are other possible outcomes on which to base allocation (graft survival, patient survival, waitlist survival), one advantage of LYFT is that it encompasses several of these metrics. Issues with the use of benefit-based allocation include predictability, and the timeframe by which benefit is measured - short timeframes favor patients with limited waitlist and post-transplant survival, and longer ones favor patients with longer survival. Regardless of the role of benefit in allocation, Dr. Stegall favors measuring it anyway, as it increases information for patients and is a robust tool for assessing outcomes and access to transplantation.

Dr. Henry countered that under a benefit-based system, older candidates would be disenfranchised, as their benefit tends to be lower than that of young candidates. He admitted that adding dialysis time (DT) and allowing candidates with less LYFT greater access to high DRI donor organs are more palatable. However, this still does not address the underlying weaknesses of the model and its use which include the following: the fact that benefit varies by timeframe (as mentioned above), there are no identifiers for cardiovascular risk, and the exclusion of race. In addition, older patients who are sicker will get only higher risk transplants, which will further worsen their outcomes, living donation is likely to decrease, and LYFT score will decrease with time on the waitlist, which will accelerate the disparity between low and high LYFT for access to kidneys over time. In addition, an average increase of $0.43 \mathrm{yr}$ of benefit per transplant seems meager in exchange for a less predictable system with methodological weaknesses that appears to substantially shift the demographics of recipients.

\section{Summary}

Solid organ transplant outcomes continue to improve in an increasingly complex environment in which recipient demand has never been higher. As donor and recipient selection criteria have changed to accommodate the increasing demand, transplant outcomes are being more strongly influenced by recipient and donor factors and that finding the right organ for the right recipient is more important than ever. Progress in more efficient and accurate donor screening methodology permits transplants from organ donors with risk factors that in the past precluded consideration. More precise and specific methods for determining and quantifying the presence of antidonor HLA antibody have resulted in innovative solutions to reduce the impact of these antibody barriers, through desensitization protocols or working around the barriers with paired donor exchanges. Improved data analyses of complex dual organ recipient outcomes are improving understanding of how to best apply combined renal/extra-renal transplants for maximal patient and graft survival. In addition, use of more robust SRTR data combined with application of advanced analytical methods are helping define the donor and recipient variables that interact and impact clinical outcomes. This has also led to donor risk indices for specific organs that improve 
our ability to forecast outcomes, thus allowing improved decision making and informed consent for prospective recipients. All of these advances also stimulate re-evaluation of current organ allocation policies.

\section{References}

1. Kucirka LM, Namuyinga R, Hanrahan C, Montgomery RA, SEgev DL. Formal policies and special informed consent are associated with higher provider utilization of CDC high-risk donor organs. Am J Transplant 2009: 9: 629.

2. GRADY D. Patients contract 2 viruses from donor in transplants. New York Times November 14, 2007.

3. Patel AM, Pancoska A, Mulgaonkar S, Weng Fl. Renal transplantation in patients with pre-transplant donor-specific antibodies and negative flow cytometry crossmatches. Am J Transplant 2007: 7: 2371.

4. Burns JM, Cornell LD, Perry DK et al. Alloantibody levels and acute humoral rejection early after positive crossmatch kidney transplantation. Am J Transplant 2008: 8: 2684.

5. Segev DL, Gentry SE, Warren DS, Reeb B, MontGOMERY RA. Kidney paired donation and optimizing the use of live donor organs. JAMA 2005: 293: 1883.

6. Montgomery RA, Zachary AA, RAtner LE et al. Clinical results from transplanting incompatible live kidney donor/recipient pairs using kidney paired donation. JAMA 2005: 294: 1655.
7. Davis CL, Feng S, Sung R et al. Simultaneous liverkidney transplantation: evaluation to decision making. Am J Transplant 2007: 7: 1702.

8. Ruiz R, BARri YM, Jennings LW et al. Hepatorenal syndrome: a proposal for kidney after liver transplantation (KALT). Liver Transpl 2007: 13: 838.

9. Campbell MC, Kotlyar DS, Brensinger CM et al. Renal function after orthotopic liver transplantation is predicted by duration of pretransplantation creatinine elevation. Liver Transpl 2005: 11: 1048.

10. Greussner R, Sutherland D, Gruessner AC. Mortality assessment for pancreas transplants. Am J Transplant 2004: 4: 2018.

11. Segev DL, Kucirka LM, NGuyen GC et al. Effect modification in liver allografts with prolonged cold ischemic time. Am J Transplant 2008: 8: 658.

12. Segev DL, Maley WR, Simpkins CE et al. Minimizing risk associated with elderly liver donors by matching to preferred recipients. Hepatology 2007: 46: 1907.

13. Schaubel DE, Sima CS, Goodrich NP et al. The survival benefit of deceased donor liver transplantation as a function of candidate disease severity and donor quality. Am J Transplant 2008: 8: 419.

14. Aвt PL, Desai NM, Crawford MD et al. Survival following liver transplantation from non-heart beating donors. Ann Surg 2004: 239: 87.

15. Lee KW, Simpkins CE, Montgomery RA, Locke Je, SEgev DL, MALEY WR. Factors affecting graft survival after liver transplantation from donation after cardiac death donors. Transplantation 2006: 82: 1683. 